\title{
MANAGEMENT OF PSORIATIC ARTHRITIS IN CHILDREN
}

\author{
Alina Murgu, Evelina Moraru, Constantin Ailioaie, Ileana Ioniuc, Monica Alexoae, Bogdan Stana \\ Gr. T. Popa University of Medicine and Pharmacy, Iasi, Romania \\ $2^{\text {nd }}$ Pediatrics Clinic, Sf. Maria Children Emergency Clinical Hospital, Iasi, Romania
}

\begin{abstract}
Psoriatic arthritis affects $15 \%$ of children with chronic rheumatic joint disease.

Aim of the study. To evaluate articular prognosis in 9 children (6-16 years) diagnosed with psoriatic arthritis and treated with different therapeutic agents, related to the degree severity of the cutaneous involvement.

Results. Sex ratio in the study group was girls/boys $=3 / 6.1^{\text {st }}$ degree relatives with psoriasis were found in $5 / 9$ cases. Poliarticular juvenile arthritis with multiple localizations (small distal hand and feet joints, radiocarpal, tibiotarsian joints) were found in 6 cases; the other 3 patients had extensive oligoarthritis (no identified HLA B27 association). None of the patients had ankyloses or severe functional motor deficiency. PASI score $<10$ was found in 4 children. Specific therapy consisted of methotrexate (2 patients) and association between methotrexate and etanercept (7 patients); All 9 children received local topical therapy. After 6-month treatment, the evaluation of patients who have received methotrexate associated with etanercept found ACR Pedi 30 response in 4 children and ACR Pedi 50 in 3 cases; PASI score was lower than 10 in 5 of these children. The 2 patients under methotrexate monotherapy showed neither ACR Pedi 30 response, nor improvement of the cutaneous symptoms after 6 months of treatment, so etanercept was added to methotrexate. The 12-month evaluation showed ACR Pedi 30 response in all 9 children; ACR Pedi 50 response in 6 children; PASI $<10$ in 7 children.

Conclusions. Early introduction of etanercept in addition to methotrexate seems to improve the prognosis of psoriatic arthritis, as well as skin disease, aspects that clearly ensure optimization of the quality of life of these patients.
\end{abstract}

Keywords: psoriatic arthritis, child, prognosis

Psoriasis is a chronic inflammatory disease that can affect persons of any age, but most of the patients are, at onset, adolescents and young adults. Its morbidity is directly related to the severity of the skin damage or systemic involvement, frequently as chronic arthropathy in $15-20 \%$ of cases, sometimes with disabling outcomes. At childhood age, the skin and joint symptoms can be found simultaneously or at a certain time interval, arthritis preceding by years the cutaneous manifestations.

The objective of the study is to assess the course of articular involvement in children diagnosed with psoriatic arthritis under various treatment regimens and in relation to the severity of skin lesions.

\section{MATERIAL AND METHODS}

The study was conducted on a group of nine patients (aged 6-16 years) diagnosed with psoriatic arthritis undergoing individualized treatment: methotrexate, etanercept and methotrexate, specific topical skin treatments. Evolution was assessed over one year, applying the ACR Pedi 30, ACR Pedi 50 response criteria for arthritis and PASI score for psoriasis.
PASI score quantifies the percentual extension of the psoriatic skin lesions (1-4) characterized by: desquamation, induration, erythema. Cutaneous affection score below 10 defines the mild-moderate form and over 10 is specific for the severe form of the disease.

The ACR Pedi 30/ACR Pedi 50 response is based on the following criteria assessing the severity of joint damage: number of swollen joints, reduced mobility and pain and/or tenderness (percentage improvement (-) or worsening $(+)$ compared to the score of the initial assessment); global assessment of severity by the physician (1-10); overall disease severity assessed by the patient (Visual Analogue Scale - VAS; score 1-10); CHAQ (Childhood Health Assessment Questionnaire) [8 fields: dressing, eating, walking, daily hygiene and other daily activities etc.; $0-3]$; ESR $(\mathrm{mm} / \mathrm{H})$ and CRP (quantitative). A $30 \% / 50 \%$ improvement since baseline for 3 out of the 6 criteria for patients under therapy defines the ACR Pedi 30 and ACR Pedi 50 response, respectively. 


\section{RESULTS}

Sex ratio was $3 / 6$ females/males. $1^{\text {st }}$ degree relatives with psoriasis were found in 5/9 patients. Polyarticular juvenile idiopathic arthritis (JIA) (small limb joints, radiocarpal, tibiotarsian) were found in 6 cases. Extensive oligoarthritis was found in 3 cases; in group age 6-12 years -2 had polyarticular JIA and 2 had oligoextensive arthritis; in 13-16 years group -4 had polyarticular JIA and 1 had oligoextensive involvement (Fig. 1). There was no HLA B27 identified association. None of the patients had ankyloses or severe functional motor deficiency. 5 out of 9 patients had psoriasis preceding JIA and 4 patients had

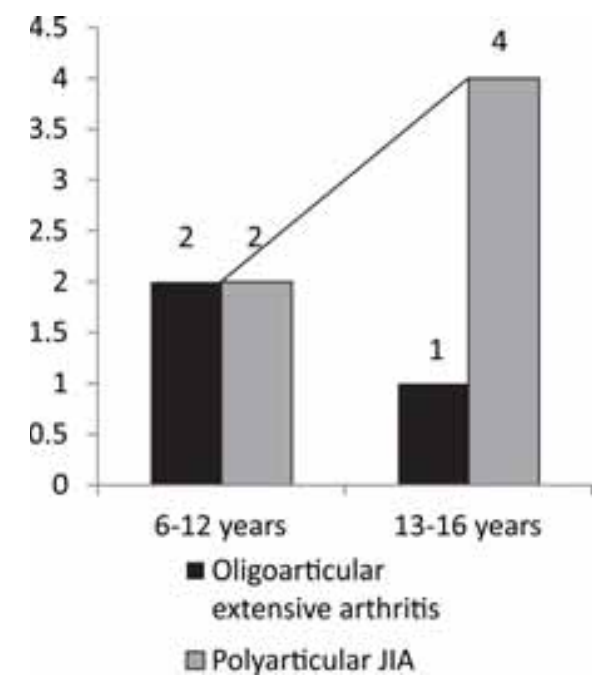

FIGURE 1. Oligoarticular and polyarticular arthritis in different age groups
JIA before psoriasis. Initial PASI score was $<10$ in 4 patients and $>10$ in 5 patients.

In our group the clinical picture was dominated by the joint pain, morning stiffness, joint swelling and dactylitis without deformation or debilitating ankylosis, functional deficit during flares, eye involvement associated with episcleritis (Fig. 2).

\section{TREATMENT}

Treatment was methotrexate monotherapy in 2 patients, who after 6 months switched to association therapy (etanercept + methotrexate). In the other 7 patients, etanercept + methotrexate therapy was instituted at baseline and continued throughout the 12 months of follow-up; all 9 patients received topical therapy.

At the 6-month evaluation, the 7 patients on etanercept + methotrexate treatment showed improvement: ACR Pedi 30 response in 4 patients and ACR Pedi 50 response in 3 patients. PASI score less than 10 was found in 5 patients. The 2 patients under methotrexate monotherapy showed neither ACR Pedi 30 response, nor improvement in the cutaneous symptoms after 6 months of treatment, so they etanercept was added to methotrexate for the following 6 months.

Evaluation after 12 months since baseline showed ACR Pedi 30 response in all 9 patients, ACR Pedi 50 response in 6 patients. PASI score less than 10 was found in 7 patients (Fig. 3, 4).
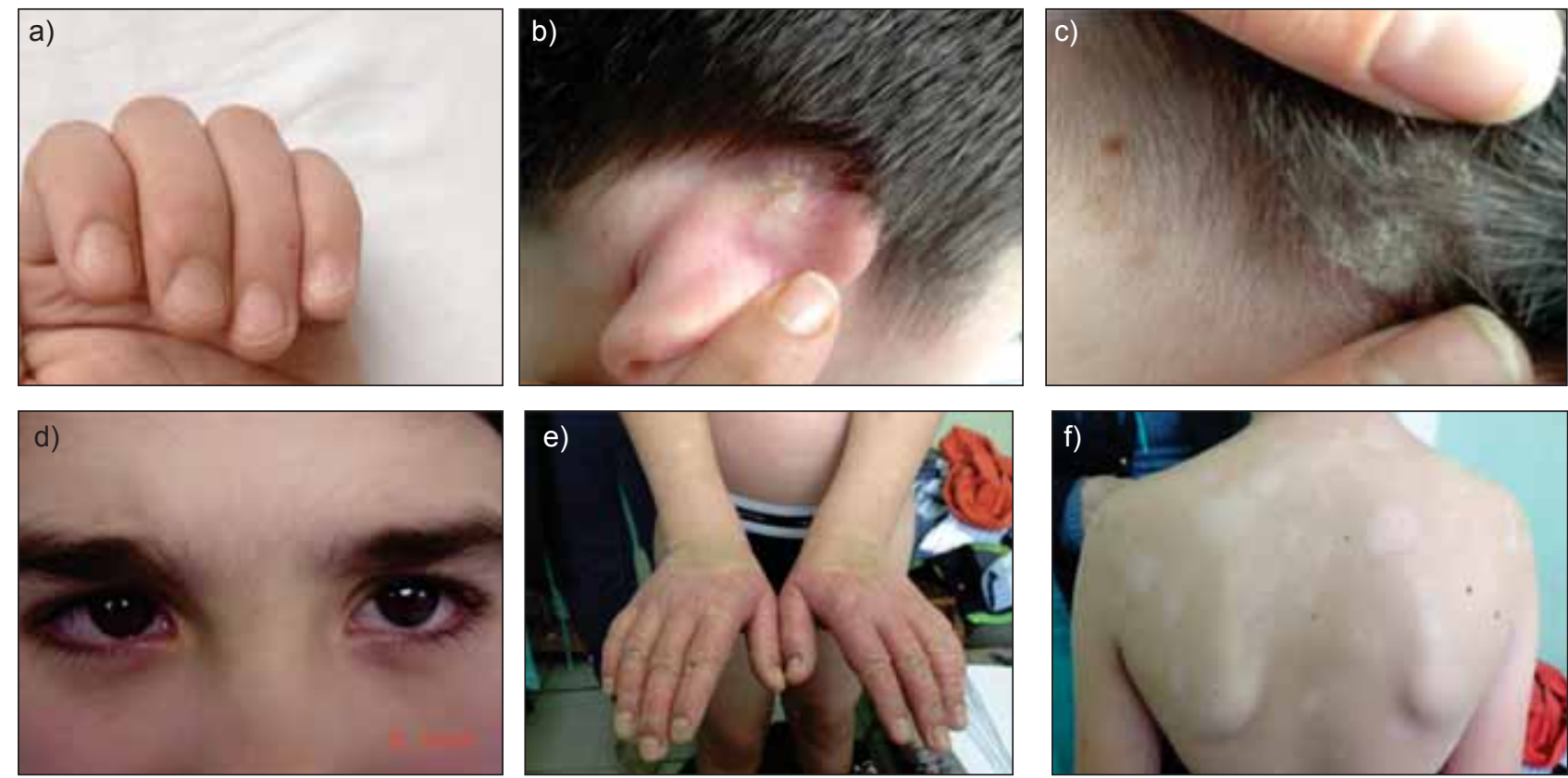

FIGURE 2. Different clinical aspects of psoriatic arthritis: a) Nail involvement; b) Retroauricular lesions; c) Scalp lesions; d) Conjunctival redness; e) Cutaneous lesions of the hands and fingers; f) Back skin depigmentations 


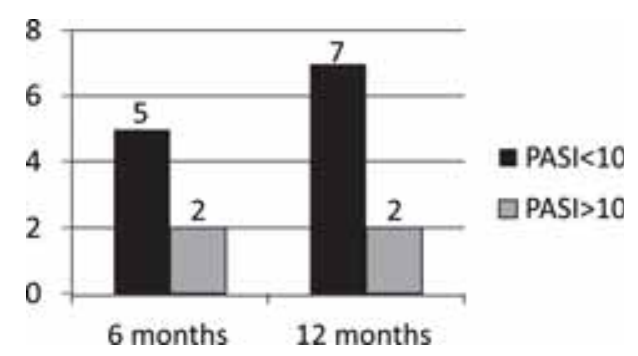

FIGURE 3. PASI score in the 9 patients

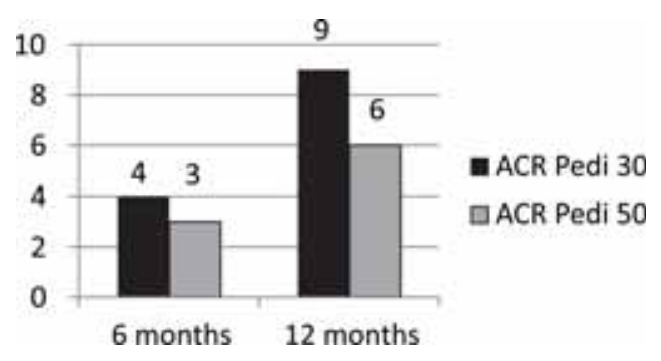

FIGURE 4. ACR Pedi 30 and Pedi 50 response in the 9 patients

\section{DISCUSSIONS}

Psoriatic arthritis is included as a subtype of JIA in ILAR (International League Against Rheumatism) classification and EULAR (European League Against Rheumatism). It has a low incidence in children in all JIA, affecting mostly adolescents and adults without a sex predisposition, although in the study group boys slightly prevailed (5). The risk of developing chronic arthropathy during psoriasis is reported differently among geographical areas $(5,8$, 15). The common key-element of the immune inflammation in both psoriasis and JIA is TNF alpha 1 A (ligand) which acts through a specific cytokine complex (IL-23, IL-17) (3) and whose activity is modulated by genetic polymorphisms SNPs (Single Nucleotide Polymorphisms) under the action of common triggers such as viral or bacterial infections $(1,2,6,7)$. These issues explain heterogeneity of both clinical expression and response to treatment with anti TNF alpha biologic agents. Although genetic involvement is known, there are not always psoriasis or other autoimmune diseases identified in relatives of patients with psoriatic arthritis (5). In the study group only 5 patients had $1^{\text {st }}$ degree relatives with psoriasis without any other type of autoimmune pa- thology in the family. Onset of articular involvement can be concomitant to skin manifestations but it also may precede or occur later than the onset of psoriasis. In our study cutaneous lesions appeared before arthritis in 5/9 cases and in 4 cases arthritis preceded the rash.

It seems that there is a correlation between the severity and extension of skin damage (PASI score) and clinical form of JIA, although it seems that the most common form is seronegative polyarthritis vs. extensive oligoarthritis or spondyloarthropathies $(10,14,16)$. Only a small percentage of children develop moderate to severe psoriasis (PASI score $>$ $10 \%$ ) frequently those look like papules or plaques on the scalp and limbs and nail damage $(5,15)$. In our group 5/9 patients had PASI score $>10$ developing both oligoarticular extensive JIA and seronegative polyarthritis, without a typical predisposition of age onset.

Initiating treatment with oral immunosuppressants like methotrexate, cyclosporine, retinoids should be done early, especially in forms with severe skin involvement (11) associated with specific topical medications. Therapeutic response must be quantified in terms of improving the two associated diseases. Patients refractory to immunosuppressants benefit from treatment with anti-TNFalpha biologic agents (such as etanercept) $(9,11,12)$ with long-term efficacy: improvement in psoriasis severity score (PASI) and JIA response (ACR Pedi 30, 50). Etanercept is the only biological agent approved to treat psoriasis in children associated with arthritis; in the children of our study group it did not induce any complications or worsening of skin disease $(4,11)$. Some studies warn that the use of biological agents other than anti TNF alpha (etanercept) in children for the treatment of inflammatory bowel disease or JIA may induce psoriasis (13).

\section{CONCLUSION}

In conclusion, early introduction of etanercept in addition to methotrexate improved outcomes, along with improving skin manifestations and optimization of the quality of life of these patients.

\section{REFERENCES}

1. Horton D.B., Scott F.I., Haynes K., Putt M.E., Rose C.D. et al. Antibiotic Exposure, Infection, and the Development of Pediatric Psoriasis: A Nested Case-Control Study, JAMA Dermatol. 2016, Feb; 152(2):191-9.
2. Li L., Fu L., Lu Y., Wang W., Liu H., Li F., Chen T. TNF-like ligand $1 \mathrm{~A}$ is associated with the pathogenesis of psoriasis vulgaris and contributes to IL-17 production in PBMCs, Arch Dermatol Res. 2014 Dec; 306(10):927-32. 
3. Windschall D., Müller T., Becker I., Horneff G. Safety and efficacy of etanercept in children with the JIA categories extended oligoarthritis, enthesitis-related arthritis and psoriasis arthritis, Clin Rheumatol. 2015 Jan; 34(1):61-9

4. Moustou A.E., Kakourou T., Masouri S., Alexopoulos A., Sachlas A., Antoniou C. Childhood and adolescent psoriasis in Greece: a retrospective analysis of 842 patients, Int J Dermatol. 2014 Dec; 53(12):1447-53

5. Murdaca G., Gulli R., Spanò F., Lantieri F., Burlando M. et al. TNF-a gene polymorphisms: association with disease susceptibility and response to anti-TNF- $\alpha$ treatment in psoriatic arthritis, $J$ Invest Dermatol. 2014 Oct; 134(10):2503-9.

6. Képíró L., Széll M., Kovács L., Keszthelyi P., Kemény L. et al. Genetic risk and protective factors of TNFSF15 gene variants detected using single nucleotide polymorphisms in Hungarians with psoriasis and psoriatic arthritis, Hum Immunol. 2014 Feb; 75(2):159-62.

7. Matusiewicz D., Koerber A., Schadendorf D., Wasem J., Neumann A. Childhood psoriasis--an analysis of German health insurance data, Pediatr Dermatol. 2014 Jan-Feb; 31(1):8-13

8. Horneff G., Burgos-Vargas R., Constantin T., Foeldvari I., Vojinovic J., Chasnyk V.G. et al. Paediatric Rheumatology International Trials Organisation (PRINTO); Efficacy and safety of open-label etanercept on extended oligoarticular juvenile idiopathic arthritis, enthesitis-related arthritis and psoriatic arthritis: part 1 (week 12) of the CLIPPER study, Ann Rheum Dis. 2014 Jun; 73(6):1114-22

9. Gladman D.D. Early psoriatic arthritis, Rheum Dis Clin North Am. 2012 May; 38(2):373-86

10. Sticherling M. Children and adolescents with psoriasis. What therapy is recommended? Hautarzt. 2012 Mar; 63(3):192-201.

11. Fraga N.A., Paim Mde F., Follador I., Ramos A.N., Rêgo V.R. Refractory erythrodermic psoriasis in a child with an excellent outcome by using etanercept, An Bras Dermatol. 2011 Jul-Aug; 86(4 Suppl 1):S144-7

12. Perman M.J., Lovell D.J., Denson L.A., Farrell M.K., Lucky A.W. Five cases of anti-tumor necrosis factor alpha-induced psoriasis presenting with severe scalp involvement in children, Pediatr Dermatol. 2012 Jul-Aug; 29(4):454-9

13. Stoll M.L., Punaro M. Psoriatic juvenile idiopathic arthritis: a tale of two subgroups, Curr Opin Rheumatol. 2011 Sep; 23(5):437-43.

14. Chiam L.Y., de Jager M.E., Giam Y.C., de Jong E.M., van de Kerkhof P.C. et al. Juvenile psoriasis in European and Asian children: similarities and differences, Br J Dermatol. 2011 May; 164(5):1101-3

15. Tuttle K.S., Vargas S.O., Callahan M.J., Bae D.S., Nigrovic P.A. Enthesitis as a component of dactylitis in psoriatic juvenile idiopathic arthritis: histology of an established clinical entity, Pediatr Rheumatol Online J. 2015 Feb 28;13:7. 